\title{
Axiomatic Design for Creativity, Sustainability, and Industry 4.0
}

\author{
Christopher A. Brown ${ }^{1, *}$ Erwin Rauch \\ ${ }^{1}$ Worcester Polytechnic Institute, Department of Mechanical Engineering, 01609 Worcester, MA, USA \\ Industrial Engineering and Automation (IEA), Free University of Bozen-Bolzano, Piazza Università 1, 39100 Bolzano, \\ Italy
}

\begin{abstract}
This paper discusses how to foster creativity and sustainability during Axiomatic Design processes, including Industry 4.0 as an example application. Creativity is generating valuable, new ideas. Innovation is making new ideas viable. This paper explains how AD theory and methods can improve the selection process in evolution-inspired creativity for formulating functional requirements and generating and selecting design parameters. FR formulation is a key to creating value in design solutions. No design solution can be better than its FRs. The FRs must capture the true, underlying essence of customer needs. In addition, an FR must define the solution space appropriately, so that all the best DP candidates are included. Suh's axioms are used to select the single best DPs from the candidates. In AD, viability is established systematically during the axiomatic decomposition and the physical integration processes. Methods for detecting poor design thinking are presented. Metrics and tests for evaluating FRs' facility for creativity and innovation are proposed. Techniques for improving FRs are proposed, decomposed, and reviewed for their compliance with the axioms.
\end{abstract}

\section{Introduction}

\subsection{Objective and rationale}

The objective of this paper is to show how creativity and sustainability can be systematically integrated into design processes using Axiomatic Design (AD) methods and applied to Industry 4.0 (I4.0). Creativity is the generation of valuable, new ideas. Innovation, often a companion term to creativity, is making new ideas viable. AD methods apply Suh's axioms to a systematic design process [1] that can make creative ideas feasible. Sustainability is essential to viability and value in engineering.

This approach is important because creativity and sustainability are essential to the development of good design solutions, although details of how to systematically include these things in the design process are not well recognized. Previous industrial revolutions have created many sustainability challenges. Perhaps I4.0 provides an opportunity for remaking production systems and initiating a green industrial revolution [2].

This is also important because not many engineers are familiar with ethics and design theories, at least formally. Unfortunately, few engineers, engineering educators, engineering administrators, and engineering students know even the first canon of engineering ethics. In addition, few engineers can name any design theories or formal design methods, nor can they appreciate how these can be systematically integrated with creativity and sustainability. The teaching of ethics in engineering schools is often limited to the minimum instruction for meeting accreditation criteria. Climate change has become widely recognized as a global crisis. Design solutions must be consistent with sustainability, if something of life as it has been known on this planet can be saved. Ethics are integral to viability and integrity in engineering design solutions.

The first canon of engineering ethics states that the safety, health, and welfare of the public must be held paramount [3]. If someone does not hold these three things of greatest value, then that individual is not doing engineering and not behaving as an engineer. Because sustainability is essential to the safety, health, and welfare of the public and the planet we all live on, it is inseparable from the first cannon. Fostering sustainability in design practices is especially important, because the future of life on this planet depends on it.

As a design theory, $\mathrm{AD}$ is exceptional because it establishes axioms for testing the viability of all kinds of design solutions and disciplines. Suh's design axioms elevate design to a scientific discipline, because it consists of a few simple, self-consistent principles that can be applied to solve a wide variety of problems [1].

Conventionally I4.0 includes applying recent technological developments to manufacturing. These developments include, artificial intelligence, Internet of Things (IoT), cyber physical production systems, and collaborative robotics. I4.0 can include more than that. Industry uses significant amounts of energy and produces waste in many forms. I4.0 should, through newly

\footnotetext{
* Corresponding author: brown@,wpi.edu
} 
available technologies and improved productivity, address sustainability.

Systematically integrating creativity and sustainability is important because design is transdisciplinary and ubiquitous. Everything people consciously interact with can involve design. For most people, design would be a secondary discipline. Everyone who seeks solutions to problems, plans or creates, anything from fine arts to zoological theories, is designing. Suh's two axioms can be applied to solve problems in everyone's primary discipline, a wide variety. Like all scientific laws, these two axioms exploit the basic, compact nature of the universe for anybody designing anything. Discussions about how to integrate ethics, creativity, innovation, and sustainability into AD are important to advancing the practice of engineering design.

A systematic approach to creativity, innovation, and ethics would be important for students and teachers of design, technology, and engineering, at all levels, from kindergarten to post doc. Currently, scientific theories and methods are introduced to students at a young age. It is indisputably important that everyone understands science, even though relatively few people become scientists. Design theories and methods are not systematically introduced to students at any age, even to engineering students. Nonetheless, everyone solves problems; therefore, we are all designers. We could all benefit from an understanding of design theory and methods. We should all embrace ethics and sustainability.

\subsection{State of the Art}

There is, of course, considerable literature on creativity and sustainability. This literature is found in many fields, including philosophy and many scientific and engineering disciplines. These concepts have been included in AD processes and discussed in a much narrower part of the literature. This part of the literature is briefly reviewed here.

According to Suh (1990, p. 9) [1], creative processes synthesize new ideas, or solutions, without prior examples, i.e., prior art. He notes two processes in design: creative and analytical. Analytical processes evaluate ideas for making design decisions, i.e., selection among ideas. AD theory states that good design solutions comply with Suh's axioms, first maintaining independence of the functional elements, and second minimizing the information content [1]. The theory states that the axioms are used in the analyses to select the best design solutions from all the candidate ideas.

In the $\mathrm{AD}$ method, design ideas, or candidate solutions, are tested against Suh's axioms at each level of abstraction, in systematic, zig-zagging decompositions, from abstract to detailed, in three or four domains. The domains are identified as customer, functional, physical, and process domains. These contain customer needs (CNs), functional requirements (FRs), design parameters (DPs), and process variables (PVs), respectively. The DPs are the physical part of the design solution. A complete design solution includes physical integration, uniting the detailed DPs into a physical model, which complies with the axioms [1].

Park [4] discussed teaching conceptual design using AD. He used open-ended design projects, done in groups. His description did not address specifics about how to develop concepts. Most decisions were made by intuitive heuristics, experience, and brainstorming. The conclusion was modest, declaring AD to be good for an objective or scientific method. Mathematical formulae were not used, and the experience of the instructor was emphasized.

Foley and Harðardóttir [5] studied manifestations of artistic creativity developed in a multidisciplinary collaboration with AD. Engineering and artistic disciplines communicate through an abstract analysis of the artistic needs, defined in terms of feelings and experiences, which become FRs. DPs are proposed, and the opinion of the collaborating artist is the test for fulfillment of the FR. The key is communication through sufficiently abstract expression of the FRs.

Four steps to the design process were presented by Suh and Sekimoto in 1990 [6], then paraphrased by Kim and Cochran in 2000 [7]. They are further paraphrased below, to be cast in the imperative for use later in this paper as FRs in a new design problem.

Table 1. Four steps in the design process.

\begin{tabular}{|c|}
\hline Design process \\
\hline $\begin{array}{c}\text { (1) Define required functions (FRs) to solve the } \\
\text { problems posed by the customer needs (CNs). }\end{array}$ \\
\hline $\begin{array}{c}\text { (2) Create ideas for solutions (DPs), maybe several } \\
\text { candidates, to fulfill each FR. }\end{array}$ \\
\hline (3) Select the best candidates for solutions. \\
\hline (4) Check complete solutions against CNs. \\
\hline
\end{tabular}

Suh and Sekimoto [6] note that each step can require iteration. These iterations can include going back to step one to redefine FRs, and to step two to create new ideas, modifying proposed solutions. Importantly, Suh and Sekimoto [6] also note that, ultimately, design solutions are represented by design equations, which relate functions and solutions. The fulfillment of each FR by a $\mathrm{DP}$, and the possible influences of others, is represented mathematically. Kim and Cochran [7] state that AD covers just the third step in Table 1, where Suh's axioms discern good and bad design solutions. They go on to state that $\mathrm{AD}$ suffers from a lack of systematic approaches to finding satisfactory candidate DPs.

The creativity step is discussed in C-K theory [8] and TRIZ [9], both of which have been integrated with AD. TRIZ proposes forty kinds of inventive concepts, gleaned from examining patents. C-K theory builds on design spaces of concepts and knowledge, exploring and expanding each to accommodate new ideas, which require new knowledge.

The cross-disciplinary journal, Sustainability, decomposes sustainability into four elements: environmental, cultural, economic, and social. All four of these can be included in design problems. Brown [3] 
formulates two basic FRs for $\mathrm{AD}$ of manufacturing processes, one to add value and the other to minimize cost. Cost includes the cost of sustainability, which is part of the first canon of engineering ethics. To hold paramount the safety, health, and welfare of the public, engineers certainly must address the environment. The cultural, economic, and social aspects of sustainability should also be included.

Beng and Omar [10] take a more detailed view about sustainability, specific to engineering design. They note three key areas that must be considered for developing sustainable products: end-of-life management, green supply chains, and sustainable manufacturing. These address the environmental component. Proper training in design for sustainability, along with a global perspective, are required for engineers. Sustainability problems require multiscale solutions, seamlessly integrated into design processes. $\mathrm{AD}$ processes have advantages. The domains in $\mathrm{AD}$ distinguish objectives or intent (FRs) from solutions and means (DPs and PVs). Decompositions in $\mathrm{AD}$, from abstract concepts to detailed solutions, enable the development of multiscale solutions that consistently embody principles for sustainability. Beng and Omar [10] conclude that including FRs for end-of-life management at the highest levels leads to design alternatives that can be profitable. The information axiom can address multicriteria problems for green supplier selection. They proposed a decomposition for sustainable product development with rules for decision-making in their three key areas.

Elaborating on integrating a manufacturing component into sustainability and $\mathrm{AD}$ processes, Poser and $\mathrm{Li}$ [11] note that clean processing can be either as a constraint (C) or an FR-DP pair. Constraints are favored, because they avoid producing anything unwanted, rather than having to find a solution to dealing with unwanted byproducts. They use toxins as an example, not producing them as a constraint, is preferable to removing them from waste streams, which requires an FR-DP pair. Taking a similar approach, Lee and Badrul [12] compare materialremoval processes. Rather than Cs, they define tolerances for energy use in the FRs and waste products in materialremoval processes. They calculate the information content based on the probability of achieving the tolerances needed to select the best material-removal process.

Brown [13] writes that I4.0 is often defined by the solutions it offers, i.e., DPs, including, cyber-physical production systems, IoT, collaborative robots, and artificial intelligence. An issue for $\mathrm{AD}$ of $\mathrm{I} 4.0$ is to understand the kinds of design problems, i.e., FRs, that are solved best by these DPs. According to Suh [14] an FR0, the top FR, for enterprises can be provide adequate return on investment (ROI). This suggests that FR children should be minimize investment and maximize return. However, these FRs only work when there are systems for maximizing or minimizing that can be DPs. The DPs for I4.0 solutions should be considered broadly, beyond the new, high-tech solutions promoted with I4.0, because other solutions might require less investment [13] and offer adequate return.
I4.0 raises new social, cultural, and economic sustainability issues. In his novel Player Piano, Kurt Vonnegut describes a highly automated and somewhat disturbing new world [15]. This fictional society is confronted with new industrial revolutions that have similarities to I4.0. Vonnegut discusses the societal, cultural, and economic consequences of the devaluation of human thought by thinking machines. Vonnegut did not, however, anticipate AD.

Potentially, the technological innovations associated with 14.0 should create increasing global wealth while, with appropriate economic and social incentives, mitigating climate change by improving energy efficiency and reducing waste [2]. Indeed, I4.0 has recently been cited as promoting energy efficiency, contributing to mitigation of climate change, and promoting sustainable energy use by industry [16].

\subsection{Approach}

Suppose that any problem can be cast as an engineering design problem. Further suppose that $\mathrm{AD}$ is the best approach to solving engineering design problems. Then $\mathrm{AD}$ is the best approach to solving any problem. The approach here consists of appropriately decomposing the problem according to procedures used in $\mathrm{AD}$ [17].

Here, as opposed to Park [4], quantification and formulae are discussed. In addition, abstracting the needs into appropriately broad FRs is used to provide a space for the design solution that encompasses creative solutions, as with Foley and Harðardóttir [5].

One problem examined here relates to creativity in $\mathrm{AD}$. Considering the four steps in the design process (Table 1), AD addresses the third, an analysis for selection of solutions from candidates. Steps one and two appear to be the most applicable for fostering creativity. Therefore, $\mathrm{AD}$ decomposition is applied here to solving the problems posed by the first two steps.

The second problem to be considered using an $\mathrm{AD}$ decomposition here relates to include sustainability into the solution of design problems. The four components of sustainability can make good CNs. However, they appear to overlap, meaning that they are not mutually exclusive, and, therefore, do not adapt well directly as FRs [18], although they could become constraints.

Finally, the problem is to understand how creativity and sustainability can be integrated into an approach to $\mathrm{AD}$ for I4.0. Freedom and dignity are elements of culture and society that must be sustained and included in the CNs. If I4.0 diminishes these things and serves to enrich further those who are already wealthy, then the economic component of sustainability will have failed as well. Just as the first industrial revolutions served to free people from much of the labor required for manufacturing, I4.0 has the potential to free people from mundane thought processes. The intellectual resources that are freed by I4.0 should be applied to enhancing our human experience. I4.0 improvements can be tied to mitigating climate change naturally, because I4.0 should seek improved ROI through increased efficiency and productivity, rather than 
increased wealth generation through increased energy use as in the first industrial revolutions.

Biologically inspired creativity is considered. Ideas are like genes, because they can be combined in different ways to create different solutions. To describe creativity, Suh [1] uses the term synthesis, which literally means combining ideas. Genetic algorithms and evolutionary computation can be used to create design solutions, by forming exhaustive combinations of selected elements and testing according to quantitative criteria, in order to determine the best [19-21]. Pollan [22] writes about substance-assisted mutation of ideas to assist in creativity, which would be followed by natural selection for survival of viable ideas.

\section{Methods}

AD decomposition processes are applied systematically for including and fostering creativity in $\mathrm{AD}$, and for integrating sustainability into AD processes. Finally, AD decomposition processes are applied to I4.0, to make it part of a green industrial revolution. These are rather abstract high-level decompositions, intended to keep a broad domain of applicability. This section presents the general methods for these decompositions in such situations. The following section, "3 Results", shows the content of the decompositions and explains how the choices were made.

\subsection{Collecting and understanding CNs}

The AD decomposition begins by collecting the CNs, which must include the needs of all stakeholders [23]. Then, an effort must be made to understand the CNs, so that the correct problem can be solved.

Proponents of innovation at a technical university encourage students to interview customers. This is a good idea, of course. However, the more important lesson for the students is how to derive the fundamental needs from all the CNs. These need to be understood adequately so that the best FRs can be formulated.

Henry Ford is supposed to have said that if he had asked people what they wanted; they would have said a faster horse. The implication is that the need was for transportation and that Ford understood this. People acquired large fortunes by fulfilling this need for transportation with cars. The further implication is that the automobile has been a great success. However, consider the fact that, worldwide, about one and a quarter million people are killed every year in traffic accidents [24]. In the UK alone the cost of health problems attributed to cars is about 6 billion pounds per year [25]. This would appear not to be sustainable, yet it has been sustained. Furthermore, Henry Ford is widely admired as a successful entrepreneur.

The CNs for economic sustainability through increased efficiencies can lead to I4.0 solutions that can help to reverse climate change by using less energy and creating less waste.

Allowing individuals to amass great wealth should not be a measure of success if it includes unpaid damage to the safety and health of the public. It is not ethical. It is not sustainable. It leads to social instability. Clearly, there must be more to assessing CNs than commercial success. It is difficult to see how the appearance of success, based on wealth, can change in the absence of systems for assigning costs to the industries that generate them. Economic sustainability cannot be independent of the sustainability of the environment, society, and culture. These are all coupled in fact and must be coupled in actual function as well. The unintended consequences of addressing one problem and creating others is a violation of Suh's axiom one.

\subsection{Developing FRs and Cs}

FR0 and constraints (Cs) are developed from the CNs [1, 26]. There are opportunities for creativity in design processes by collecting CNs from all the stakeholders. Fundamental needs of stated CNs should be understood and appropriately formulated into FRs.

The technique of "five whys", used by Toyota [27] to identify the root causes of a problem in production, could be applied to CNs, in order to identify and understand the fundamental needs. With this understanding, a more useful FR for fostering creative solutions might be formulated.

If it is not possible to think of several DPs that can satisfy an FR, then maybe the FR is too confining and should be changed. The region between the customer and physical spaces is where the functional space is located. This region can be a continuum. The closer FRs are to the physical space, the smaller is the solution space for that FR. The more physical the FR is, the less solution-neutral it is, and the smaller the solution space for that FR.

FRs must be developed to leave the largest possible space for the physical solution. This is intended to allow for new solution ideas. This is another opportunity for creativity. An FR that is lacking in solution neutrality can constrain the solution unnecessarily. If the interpretation of the potential consumer's self-assessed need for a faster horse had been taken literally, then the problem was to develop faster horses. This was the response for centuries previously.

The internal combustion engine and the development of metals' technologies facilitated a new, disruptive solution to the transportation problem, if it was recognized as such, and an enlarged solution space could be created. Traditional FRs had to be adjusted to exploit new solution spaces. I4.0 can be seen similarly. New production technologies can enlarge solution spaces. FRs must be adjusted to go beyond the spaces that only allowed solutions enabled by previous technologies. FRs that might once not have been considered because they were thought to be unrealistic, could now be achievable. In the decomposition, FR child elements must be collectively exhaustive and mutually exclusive (CEME) decompositions of their parents [17]. CEME decompositions comply with Suh's axioms. If the children are not mutually exclusive with respect to each other, then independence is not maintained. If children are not collectively exhaustive with respect to the parent, then 
some part of the solution has been lost, the probability of success is diminished, and the information content is not minimized, violating Suh's axiom two. Themes, like energy and time, decomposed into kinds of energy and segmentations of time, can help to verify that decompositions are CEME.

One difference between FRs and Cs is that FRs need to be mutually exclusive with respect to each other, whereas Cs might not be separable from at least some of the FRs.

Another difference is that FRs require DPs to fulfill them. To keep the solution simple, FRs should be kept to the minimum required to satisfy the CNs. Therefore, Cs are favored over FRs for meeting $\mathrm{CNs}$, however $\mathrm{Cs}$ restrict the solution space. If the solution space becomes over-constrained, then a solution might not exist in this space.

Clearly, it is better not to create waste as a byproduct, which favors dealing with the environmental aspects of sustainability as constraints. However, this constraint might overly restrict the process options, possibly leaving no options that do not violate the axioms. Then, the treatment of the waste could be added as another FR.

\subsection{Synthesizing, selecting DPs, and decomposition}

Synthesizing DPs to fulfill FRs is an important creative step. A method for solving seemingly unsolvable large problems is to decompose them into many, solvable smaller problems, then to integrate these into the solution of the larger problem.

Zigzagging decomposition between FRs and DPs at progressively more detailed levels should continue until the solution is obvious [1]. At the upper levels, with less detail, DPs might just restate FRs. If FRs are "provide X", DPs can be systems, devices, or mechanisms that provide $\mathrm{X}$. This might seem to lack value, except that it helps to categorize and define independent branches that follow specific themes. Qualifiers, like mechanical or electrical, specify and better define the theme. Different themes and qualifiers can be attempted. Genetic algorithms [21] can be used to attempt and test all the combinations against the constraints and Suh's first axiom, then rank them with Suh's second axiom, if there are enough options to merit this approach.

To solve design problems, eventually solution specifics are needed at the lower levels. Zigzagging decomposition can progress to levels where solutions to detailed FRs are obvious. This way, decompositions foster creativity by building frameworks for many small creative steps, rather than fewer, huge creative leaps. This is good, when it works. However, decomposition processes do not always arrive at this happy conclusion.

Perhaps the solution does not exist yet. A solution could require new technology. The decomposition should assist in identifying missing components. The new technology might be developed by further decomposition and understanding the problem at fundamental levels.

Decomposition processes can restrict solution spaces. To foster creativity, solution spaces should be kept as large as possible. At each step, it is good to have several candidate DPs for each FR. If not, then maybe themes and qualifiers on parent DPs should change, and maybe FRs should be changed. This might be required for synthesizing appropriate DPs.

Once several candidates have been identified, then the task is to name the best choice. Cs should be applied first, which might eliminate some candidates. If Cs eliminate too many or all DP candidates, then this could be an over-constrained approach to the problem. Maybe a new decomposition theme should be found. To enlarge the design space, some constraints could be changed to FRs. After applying Cs, Suh's axioms are applied in the usual manner to remaining candidate DPs, in order to select the best one. In the process of applying axiom one, the specific detailed solutions at the lower level need to be inherited to the upper levels, with the resulting coupling thereby reflected at these upper levels [28].

In summary, creativity is fostered by decomposing until the solution is obvious. This should provide small creative steps, which should have simple, obvious candidate solutions. The best DPs are reduced by applying the Cs and axiom one, and then ranking by axiom two. This process can combine ideas, like genes, at the most detailed level, which blend, or integrate, functionally, or are synthesized into larger creative solutions. Genetic algorithms can be used to investigate different functional combinations of detailed genes of ideas from different branches [19-21]. These new functional configurations solve larger problems at higher levels of abstraction. The next step is the physical integration of detailed DPs into a complete solution.

\subsection{Physical Integration of the DPs}

Physical integration can be another opportunity for creativity in the configuration of individual DPs into complete entities. Physical integration does not need to follow the path of the functional-physical decomposition, and generally does not. Certain physical elements need to be materially connected or supporting to achieve functionality. DPs should be combined into sub-systems and systems to achieve desired functionalities. This process resembles the decomposition process, except in reverse. Multiple physical integration configurations can be considered. Again, genetic algorithms and an evolutionary approach to creativity [19-21] can be used to evaluate all the combinations, by applying constraints and Suh's first axiom, possibly eliminating some combinations, and then by ranking those remaining with Suh's axiom two, to select the best integration solution.

A physical integration matrix, showing physical DPDP interactions, is useful to evaluate Suh's first axiom and avoid unwanted interactions. It can also assure that there are interactions where they are required.

\subsection{Sustainability and 14.0}

Metrics for the success of I4.0-related FRs need to be based on improved efficiencies in energy utilization, productivity, and waste reduction. Implementation of new 
technologies in I4.0 cannot be sustainable if the metrics are based on shortsighted energy use and waste production. The earth is reaching the limits of its tolerance for non-sustainable activities. For survival, society needs to impose costs on energy use and waste production that are commensurate with the actual damage to the environment. I4.0 needs to rise to this challenge of producing wealth while preserving health. I4.0 needs to include product design, in order to design products for production and systems for use by I4.0. The technological resources of I4.0 can be used to address all products, processes, transportation, communication, and systems. This could make it a system for sustainability for all human activities.

\subsection{Representation of Design Solutions and Metrics}

Methods need to include design-solution representations and metrics. Without a representation, there is no design. Without a measurement of the level of success of a design solution, according to Lord Kelvin's legendary pronouncement about measurement, design solutions cannot be improved. Representations of design solutions commonly include information required to manufacture solution, a solid model with dimensions and tolerances, and a bill of materials. The design intent, FRs, is not commonly included in design solution representations. This makes improvement unnecessarily uncertain and replete with unintended consequences. Unless design intents are linked to design solutions in representations, changes in the design solution introduce uncertainty in amended functions. FRs record design intent and are a necessary pre-physical step in design thinking. Complete design representations could also include evidence of creative struggles by capturing all candidate DPs and the reasons for not selecting them. Evidence of creativity, innovation, ethics, and sustainability should be evident in complete representations of design solutions.

\section{Results}

The result of creative, sustainable AD is the beginning of a decomposition to address the fundamental needs. The fundamental customer, or societal, need is for sustaining the environment, cultures, economies, and societies. There are undesirable aspects of these entities that should be improved, rather than sustained. "Sustain" might not be the best term; however, these improvements and phraseology are left for discussion in other forums.

Table 2 shows the initial draft of a decomposition, intended for designing decompositions to produce creative design solutions consistent with sustainability through I4.0. Novice users of AD often mistakenly begin with an FR0 to design some artifact, when what they mean to do is to design something that will function like that artifact, e.g., a bicycle. The FR0 in Table 2. is intentionally about design. DP0 is a design system. The children in Table 2 follows the theme outlined in Table 1, and they are collectively exhaustive in that regard. There are more details in Table 2, including the developments in the methods section for creativity.

At this level the proposed DPs are appropriately abstract. Critically speaking, they might appear to add little to a design solution. Nonetheless, they clearly define specific, mutually exclusive components of the solution.

Table 2. Upper-level decomposition of design for sustainability (I4.0).

\begin{tabular}{|l|l|}
\hline \multicolumn{1}{|c|}{ FR } & \multicolumn{1}{c|}{ DP } \\
\hline $\begin{array}{l}\text { FR0 Design for } \\
\text { sustainability (I4.0) }\end{array}$ & $\begin{array}{l}\text { DP0 Creative design } \\
\text { system for sustainability }\end{array}$ \\
\hline $\begin{array}{l}\text { FR1 Develop } \\
\text { appropriate CNs }\end{array}$ & $\begin{array}{l}\text { DP1 Fundamental CN } \\
\text { development method }\end{array}$ \\
\hline $\begin{array}{l}\text { FR2 Constitute suitable } \\
\text { Cs }\end{array}$ & $\begin{array}{l}\text { DP2 Suitable C } \\
\text { constituting method }\end{array}$ \\
\hline $\begin{array}{l}\text { FR3 Formulate } \\
\text { satisfactory FRs }\end{array}$ & $\begin{array}{l}\text { DP3 FR formulation for } \\
\text { large solution spaces }\end{array}$ \\
\hline $\begin{array}{l}\text { FR4 Create ideas for } \\
\text { solutions, DPs }\end{array}$ & $\begin{array}{l}\text { DP4 Creative solutions } \\
\text { for multiple DPs } \\
\text { (iterate 1) }\end{array}$ \\
\hline $\begin{array}{l}\text { FR5 Select the best } \\
\text { solutions, DPs }\end{array}$ & $\begin{array}{l}\text { DP5 Selection method } \\
\text { on Cs and Suh's axioms }\end{array}$ \\
\hline $\begin{array}{l}\text { FR6 Integrate DPs for } \\
\text { complete solution }\end{array}$ & $\begin{array}{l}\text { DP6 Physical } \\
\text { integration method }\end{array}$ \\
\hline
\end{tabular}

The decomposition is full lower-triangular, with sequential coupling, because successful completion of each FR depends on satisfying the previous one [29]. Usually there is no need to iterate if the correct sequence is followed. Here, iteration is required if solution spaces are small, because CNs and FRs might not be sufficiently fundamental, or Cs are overly restrictive. The need to iterate is indicated by the inability to create multiple solutions.

Metrics and tests for evaluating the degree of success in fulfilling FRs should be selected when FRs are defined. Only with metrics can a DP be fully and truly evaluated for its appropriateness. Complete evaluations of DPs should include quantitative indications of sustainability, as well as of their ability to fulfill FRs. In this regard, there could be two components and two sorts of design equations containing DPs, one each for functionality and sustainability. Metrics can also be used to test for CEME in decomposition equations.

\section{Discussion}

AD provides several possibilities for fostering creativity, the synthesis of good, new ideas. Creative opportunities begin by collecting CNs from all the stakeholders [1,23].

$\mathrm{CNs}$ require fundamental interpretation to formulate FRs and Cs $[1,26]$. A design solution can be no better than the FRs [1]. Because of this, FRs are key to optimizing value in design solutions. FRs must capture the true, underlying essence of CNs. Five whys, as used in troubleshooting in lean manufacturing, can be used for getting to the root of design problems and assisting in creative FR formulation. FRs can be thought of as existing 
on a continuum that extends from the CNs to the DPs. Moving FRs away from physical solutions and toward fundamental CNs can help to enlarge the design solution space. Decompositions should be pursued to finer and finer details, until the creative steps are sufficiently small to be obvious. A good decomposition process is essential in this approach to creativity.

The fundamental nature of Suh's axioms are to establish viability through a kind of functional modeling or testing for good solutions, i.e., adjustable, controllable, avoiding unintended consequences, and robust. Innovation can be advanced by application of Suh's axioms to functional-physical decompositions and physical integrations.

Sustainability can be derived naturally from the first canon of ethics for engineers: hold paramount the safety, health, and welfare of the public. Importantly, I4.0 suggests new technologies that can fulfill FRs that previously could not be done, because there were no physical solutions available. Properly executed, I4.0 provides opportunities for achieving sustainability in a fractal-like manner. This means that, at all levels of manufacturing processes and systems, there is a selfsimilar pattern of using new technologies to improve productivity and reduce waste. I4.0 cannot truly address sustainability while fostering the current trend in the US of concentrating more wealth in the hands of fewer people. This has led to unethical management of wealth and power by climate change deniers, who ignore sustainability.

I4.0 has the potential to eliminate jobs that underutilize intellectual capacity. With proper training, newly available intellectual capacity can be used to advance sustainability and reverse climate change. Highquality education needs to be universally available. Particularly in the US, industry uses engineers, extracting value from their work, without contributing to the high cost of their undergraduate education. Foreign-educated engineers can enter the US workforce, with documentation for legal immigration, without having to repay the crushing debt acquired by many US engineers during their undergraduate education. Access to education should be based only on aptitude and not on ability to pay. Well-trained, ethical engineers are required to reverse climate change. Any society that discriminates on anything besides aptitude will underperform. All human potential should be brought to the rescue of the environment and the enhancement of sustainability. Systems that permit amassing of wealth at the cost of sustainability should not be allowed.

Complete representations of design solutions should include design intent, metrics, and logical paths leading to creative solutions. These records are more elaborate that those currently in common use. They provide for more sophisticated assessments of the design solutions and also can facilitate creativity and advance sustainability by providing guidance for future design development through strong knowledge management. Steps in the decomposition should be retained for future reference, including CNs, FRs, candidate DPs and the reason for their rejection or retention. Industry is losing value in the design process by missing this opportunity.

\section{Conclusions}

1. AD provides several possibilities for fostering creativity, including understanding fundamental needs of the customers and stakeholders, defining satisfactory FRs, and creating multiple DP candidates for selection. The latter can be achieved by decomposing until the solution is obvious.

2. Viability for advancing innovation can be achieved by application of Suh's axioms to functional-physical decompositions and physical integrations.

3. Properly executed, Industry 4.0 provides opportunities for achieving sustainability in a fractallike, self-similar, multiscale manner.

4. Representations of design solutions, including FRs for design intent, metrics for FRs and DPS, and logical paths leading to creative solutions, with alternative DPs, can advance sustainability and provide valuable guidance for future design works. Design software should include these features.

5. Definitions of Industry 4.0 should include FRs, i.e., design intent, emphasizing its potential to address sustainability, including engineering ethics, the safety, health and welfare of the public, and climate change

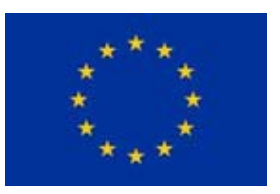

This project has received funding from the European Union's Horizon 2020 research and innovation program under the Marie Skłodowska-Curie grant agreement No. 734713.

\section{References}

1. Suh, N.P., 1990. The principles of design. Oxford University Press.

2. White, D.F., 2002. A green industrial revolution? Sustainable technological innovation in a global age. Environmental Politics, 11(2):1-26.

3. Brown C.A., 2011. Axiomatic design for understanding manufacturing engineering as a science. In Proceedings of the 21st CIRP Design Conference.

4. Park, G.J., 2014. Teaching conceptual design using axiomatic design to engineering students and practitioners. Journal of Mechanical Science and Technology, 28 (3): 989-98.

5. Foley, J.T. and Harðardóttir, S., 2016. Creative Axiomatic Design. Procedia CIRP, 50:240-45.

6. Suh, N.P. and Sekimoto, S., 1990. Design of thinking design machine. CIRP Annals, 39(1): 145-48.

7. Kim, Y.S. and Cochran, D.S., 2000. Reviewing TRIZ from the perspective of axiomatic design. Journal of Engineering Design, 11(1): 79-94.

8. Le Masson, P., Weil, B. and Hatchuel, A., 2017. Design theory: Methods and organization for innovation. Springer.

9. Savransky, S.D., 2000. Engineering of creativity: Introduction to TRIZ methodology of inventive problem solving. CRC Press. 
10. Beng, L.G. and Omar, B., 2014. Integrating axiomatic design principles into sustainable product development. International Journal of Precision Engineering and Manufacturing-Green Technology, 1(2): 107-17.

11. Poser, H. and Li, W. eds., 2008. The Ethics of Today's Science and Technology: A German-Chinese Approach (Vol. 17). LIT Verlag Münster.

12. Lee, G.B. and Badrul, O., 2014. Optimization for sustainable manufacturing based on axiomatic design principles: a case study of machining processes. Advances in Production Engineering \& Management, 9(1): 31 .

13. Brown, C. A., 2019. Axiomatic Design for Products, Processes and Systems. In Dominik T. Matt, Vladimir Modrak, Helmut Zsifkovits (Eds.). Industry 4.0 for SMEs Challenges, Opportunities and Requirements. Basingstoke: Palgrave Macmillan (to be published in 2019), ISBN 978-3-030-25425-4.

14. Suh, N. P., 2001. Axiomatic design: advances and applications. MIT Pappalardo Series in Mechanical Engineering.

15. Vonnegut, K., 1952. Player Piano. Dial Press.

16. Fritzsche, K., Niehoff, S., and Beier, G., 2018. Industry 4.0 and Climate Change-Exploring the Science-Policy Gap. Sustainability, 10(12): 4511.

17. Brown, C.A., 2011. Decomposition and prioritization in engineering design. In Proceedings of the 6th International Conference on Axiomatic Design (p. 41).

18. Brown, C.A. and Henley, R., 2016. Metrics for Developing Functional Requirements and Selecting Design Parameters in Axiomatic Design. Procedia CIRP, 53:113-18.

19. Bäck, T., Fogel, D.B. and Michalewicz, Z. eds., 2018. Evolutionary computation 1: Basic algorithms and operators. CRC press.

20. Gabora, L. and Kaufman, S.B., 2010. Evolutionary approaches to creativity. The Cambridge handbook of creativity, pp.279-300.

21. Marczyk, A., 2004. Genetic algorithms and evolutionary computation. The Talk Origins Archive: http://www. talkorigins/faqs/genalg/genalg. html.

22. Pollan, M., 2002. The botany of desire: A plant's-eye view of the world. Random House.

23. Thompson, M.K., 2013. Improving the requirements process in Axiomatic Design Theory. CIRP Annals, 62(1):115-18.

24. Association for safe international road travel, accessed 21/09/2019, https://www.asirt.org/safetravel/road-safety-facts/

25. Shelby Rogers, 2018, UK's Car Pollution Problem Costs £6 Billion a Year in Health Damages, Interesting Engineering, accessed 21/09/2019, https://interestingengineering.com/uks-carpollution-problem-costs-6-billion-a-year-in-healthdamages
26. Thompson, M.K., 2013. A classification of procedural errors in the definition of functional requirements in Axiomatic Design theory. In Proceedings of the 7th International Conference on Axiomatic Design (ICAD 2013).

27. Serrat, O., 2017. The five whys technique. In Knowledge solutions (pp. 307-10). Springer, Singapore.

28. Brown, C.A., 2014. Axiomatic design of manufacturing processes considering coupling. In Proceedings of ICAD2014 the Eighth International Conference on Axiomatic Design.

29. Brown, C.A., 2006. Kinds of coupling and approaches to deal with them, In Proceedings of ICAD 2006 -- The fourth International Conference on Axiomatic Design, Firenze, paper 15. 